\title{
TARMED-Evaluation EFK - was wollte der TARMED erreichen?
}

Ernst Gähler ${ }^{a}$, Urs Stoffel ${ }^{b}$, Anton Prantlc,

Franziska Zoggd

a Dr. med., Vizepräsident der FMH, Verantwortlicher Tarife und Verträge

b Dr. med., Co-Präsident der Konferenz der Kantonalen Ärztegesellschaften (KKA)

c Dipl. Masch.-Ing., Direktionspräsident Ärztekasse

d Dr. med., Vorstandsmitglied Hausärzte Schweiz
Korrespondenz:

Dr. med. Ernst Gähler Tarifdienst FMH

Elfenstrasse 18

CH-3000 Bern 15

Tel. 0313591111

Fax 0313591112
Die Eidgenössische Finanzkontrolle (EFK) evaluierte seit Juni 2008 die TARMED-Tarifstruktur. Die Ärzteschaft stand dem Projekt «Evaluation TARMED» der EFK von Anfang an positiv gegenüber und beteiligte sich daran. Anhand von elf Fallbeispielen erarbeitete INFRAS im Auftrag der EFK die Datenbasis zur Abbildung der Kostenrealität. Die überprüften Fallbeispiele betreffen die medizinischen Fachgesellschaften der Hausärzte (SGAM, SGIM), der Gynäkologen (SGGG), der Ohren-Nasen-Hals-Ärzte (SGORL) sowie der Augenärzte (SOG). INFRAS hat mittels Expertengesprächen unter Einbezug der beteiligten Tarifpartner inklusive Fachgesellschaften und der Ärztekasse die Fallbeispiele evaluiert und differenziert und umfassend analysiert - mit beeindruckendem Resultat! Des Weiteren unterstützten vonseiten der Ärzteschaft Experten der FMH und der Konferenz der Kantonalen Ärztegesellschaften (KKA/CCM) die EFK bei Fragen zur Kostenneutralität und zur Tarifstruktur.

Die Analyse und die daraus gewonnenen Resultate sind nachvollziehbar. Was aber im Schlussbericht

\section{Der Evaluationsbericht der Eidgenössischen Finanzkommission (EFK) zeigt viele ausge- sprochen positive Resultate der partner- schaftlich entwickelten Tarifstruktur TAR- MED auf:}

- 26 veraltete kantonale Tarife sowie der UV/MV/IVTarif konnten mit einem Schlag durch die Tarifstruktur TARMED abgelöst werden. Grundlegende korrigierende Eingriffe waren bis heute nicht nötig.

- Drei der fünf Fragestellungen der EFK konnten positiv beantwortet werden, so die verbesserte Transparenz, die kostenneutrale Einführung und die Kostenrealität der Tarifstruktur.

- Die elf von INFRAS evaluierten Fallbeispiele und damit der Kern und die Grundlage der faktenbasierten Evaluation zeigen auf, dass die Kostenrealität in fünf Fallbeispielen als eher zu tief und lediglich in drei Fallbeispielen als eher zu hoch einzustufen ist. Die Breite der Analysen, aber auch die Fülle des analysierten Datenmaterials zur praktischen Tarifanwendung ist beeindruckend. Die weitgehend wegweisende Evaluation der INFRAS klärt nicht nur die Frage der Kostenrealität, sondern zeigt auch, welcher Aufwand für eine sachgerechte Tarifierung betrieben werden muss. Die fünf als zu tief eingestuften Fallbeispiele daraus gemacht wird, ist aufgrund der faktengestützten Ergebnisse der INFRAS-Analysen nicht wiederzuerkennen. Die Schlussfolgerungen und insbesondere die Empfehlungen sind vorwiegend politische Aussagen, ohne faktenbasierte Relevanz. Der Bericht der EFK ist deshalb für die Ärzteschaft in der vorliegenden Form nicht akzeptabel und muss zur Überarbeitung zurückgewiesen werden!

Der in «süffigem» Ton abgefasste Schlussbericht legt ausserdem den Verdacht nahe, dass die Schlussfolgerungen wohl schon vor der Evaluation feststanden. Insgesamt sind alle Beteiligten enttäuscht, dass aus dieser mit grossem Aufwand und erheblichen Ressourcen durchgeführten Studie substantiell ein so unbefriedigendes Fazit resultiert. Der Schlussbericht der EFK ist für uns ein Lehrbeispiel dafür, dass man, trotz Einbindung der Ärzteschaft in die Evaluation, diese letztlich nicht ernst nimmt.

Der Schlussbericht der EFK wird zusammen mit dem Beibericht der FMH am 23. November 2010 veröffentlicht werden.

bilden über 50\% des gesamten TARMED-Umsatzes der Arztpraxen ab!

- Die Analyse und die Antworten auf die Fragestellungen bezüglich Transparenz, kostenneutraler Einführung und Abbildung der Kostenrealität lassen die klare Schlussfolgerung zu, dass der TARMED mit Sicherheit keinen volkswirtschaftlichen Schaden verursacht.

Der Bericht dokumentiert auch deutlich die von der Ärzteschaft seit langem monierten Schwachstellen der Tarifstruktur:

- Die vom Preisüberwacher angeordnete Halbierung der letzten fünf Minuten der Konsultationstaxe hat erhebliche Auswirkungen für die Grundversorger und stellt diese ungewollt schlechter als die Spezialisten. Damit wird klar, dass mit den Eingriffen an der Tarifstruktur einzig die Verhältnisse der Spezialitäten unter sich, nicht aber die Kosten beeinflusst werden. - Von einzelnen Tarifpartnern vorgängig geforderte Kostenlimitierungen für die Revision der Tarifstruktur verhindern eine sachgerechte und betriebswirtschaftliche Weiterentwicklung und Revision des TARMED. Die FMH und $\mathrm{H}+$ als Vertreter der Leistungserbringer haben deshalb die Revision der Struktur selber in Angriff genommen. 


\section{Antworten auf Fragen zum TARMED}

Ernst Gähler
Folgende Experten nehmen zu Fragen des TARMED Stellung: Dr. med. vet. Markus Dürr, ehemaliger Präsident der GDK, (Sicht der Kantone); Prof. Dr. iur. Urs Saxer, Rechtsberater der KKA, (Gewaltentrennung Stellung des Preisüberwachers); Dr. oec. HSG Willy Oggier, Gesundheitsökonom, (TARMED aus gesundheitsökonomischer Sicht); lic. iur. Peter Meier, ehemaliger Rechtsberater der KKA, (Zuständigkeiten für Tarifstruktur - KVG-Tarife).

\section{Frage an alt Regierungsrat Dr. Markus Dürr}

Sie waren während der ganzen Einführungsphase und bis vor einem Jahr Präsident der Gesundheitsdirektorenkonferenz. Wir meinen, die Empfehlungen der EFK laufen schliesslich auf eine vollständige Beschneidung der kantonalen Hoheit heraus - Tarifstruktur und Preise werden durch Einsitznahme des Preisüberwachers und Tarifierungsrecht des Bundesrates sowie Unsetzung des Art. 59c KVV nationalisiert - die restlichen Komponenten eines von den Kantonalregierungen zu genehmigenden Tarifvertrages schaffen es nicht einmal, in der Evaluation erwähnt zu werden. Was bedeuten die Empfehlungen der EFK, dem Bund im TARMED mehr Kompetenz und Eingriffsmöglichkeiten zu geben, für die Kantone?

Dürr: Mit dem Projekt TARMED sollte ein neuer Arzttarif eingeführt werden, der betriebswirtschaftlich korrekte Entschädigungen gewährleistet. Ein Ziel war es auch, die intellektuelle Leistung des Arztes gegenüber den technischen Leistungen aufzuwerten; insbesondere sollten der Allgemeinmediziner, der Psychiater und der Kinderarzt gegenüber den Spezialisten aufgewertet werden. Das waren hehre, gerechtfertigte Ziele, die aber leider nur teilweise erreicht wurden. Durch die von der Bundespolitik aufgepfropfte Kostenneutralität, die einen Widerspruch zu einem betriebswirt-

\section{«Bitte keine Bundesexperimente zulasten der Kantone»}

schaftlich gerechneten Tarif darstellt, kam es zu heftigen Auseinandersetzungen innerhalb der Ärzteschaft (die ich als neutraler Beobachter zeitweise miterlebt habe) und die schliesslich in Kraft gesetzte Tarifstruktur ist eher das Produkt der Kräfteverhältnisse oder des Engagements einzelner Disziplinen unter der Ärzteschaft als ein korrekter betriebswirtschaftlicher Tarif. Auch sind die dem Patienten übergebenen TARMEDRechnungen alles andere als übersichtlich und selbsterklärend, kürzlich konnte in einem mir bekannten Fall die behandelnde Praxis auf Nachfrage keine Auskunft geben, auf welche von zwei in kurzem Abstand gestellten Diagnosen sich die Rechnung beziehe!

Auch die Weiterentwicklung des Tarifs ist nicht gewährleistet, es braucht Strukturen, die zu einer Entscheidung führen bei Unstimmigkeiten. Die Kantone haben unterschiedliche Tarife pro Kanton in Kauf genommen, allerdings sollten nach einer «Konvergenz-

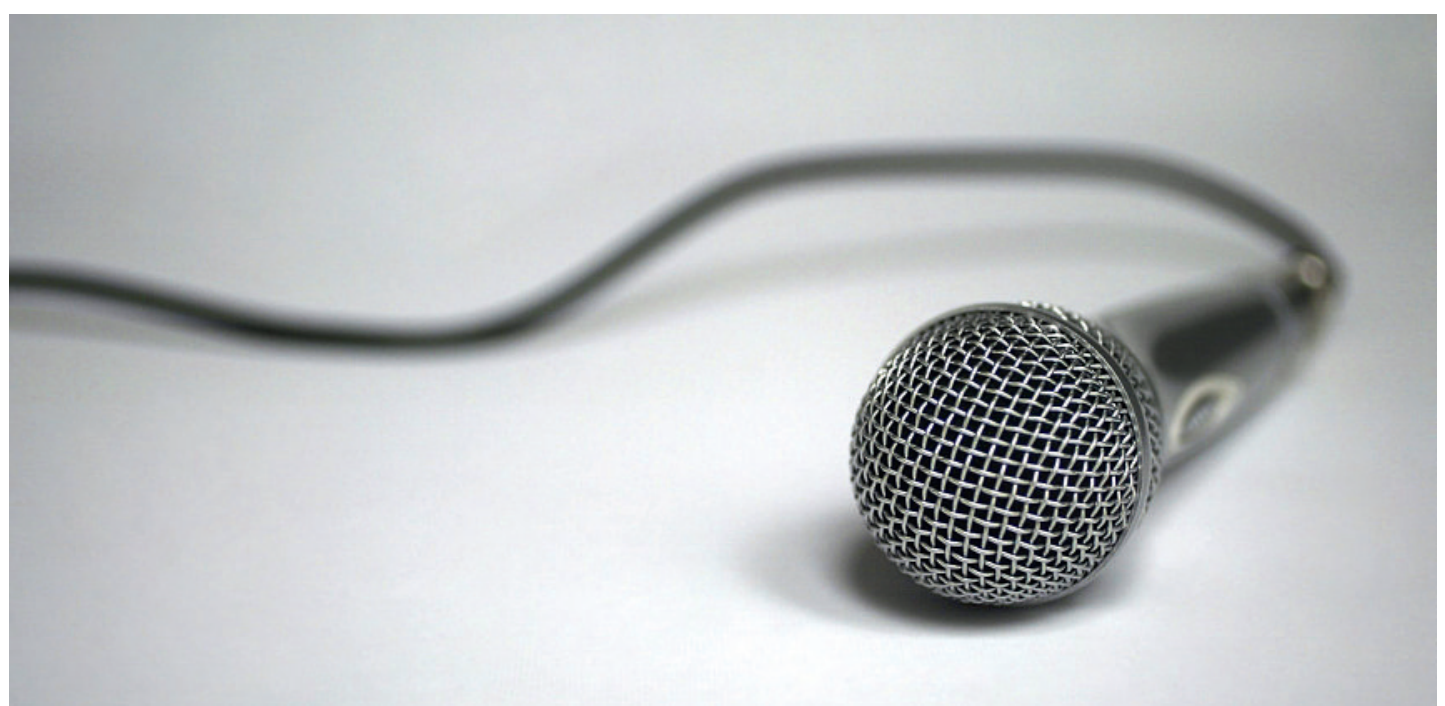

Vier Experten haben die Fragen von Ernst Gähler zu unterschiedlichen Aspekten des TARMED beantwortet: Markus Dürr, Urs Saxer, Willy Oggier und Peter Meier. 
phase» die kantonal unterschiedlichen Taxpunktwerte angeglichen werden. Stattdessen wurden die schon vorher herrschenden Differenzen zwischen den Tarifen der Kantone zementiert. Trotzdem war nie die Rede davon, dass der Preisüberwacher und/oder andere Bundesstellen in die Tarifhoheit der Kantone sollten eingreifen können. Einzig die Tarifstruktur muss national einheitlich und deshalb vom Bundesrat genehmigt sein. Die Vorschläge der EFK, wonach der Bundesrat Tarife zwischen den Vertragspartnern genehmigen soll, widersprechen dem KVG und überhaupt den Gepflogenheiten unserer föderalistischen Ordnung. Es ist nach wie vor so, dass nach Verfassung die Kantone zuständig sind für die Organisation und Umsetzung des Gesundheitswesens. Der Bund kann einzig im KVG gewisse, die Hoheit der Kantone nicht zentral unterlaufende Regelungen treffen. Alles andere fordert die Opposition der Kantone heraus, und hätten wir die Verfassungsgerichtsbarkeit, wären schon mehrere bisherige Regelungen im KVG angefochten worden. Der TARMED muss weiterhin effizient «gepflegt» werden können, momentan ist die Tarifpflege aber weitgehend blockiert. Es muss die bisherige Organisation durch eine zielführende abgelöst werden, ähnlich der Regelung der DRGs im Spitaltarif. Im Sinne einer subsidiären Kompetenz des Bundes, bei Scheitern nötiger Tarifstrukturanpassungen durch die Tarifpartner, halte ich die Empfehlung der EFK für angemessen, aber bitte keine Bundesexperimente zulasten der Kantone.

\section{Frage an Prof. Dr. iur. Urs Saxer}

Die EFK schlägt vor, den Preisüberwacher (PüW) in TARMED Suisse Einsitz nehmen zu lassen und ihm quasi ein direktes Eskalationsrecht an den Bundesrat zu geben. Ist dies im Preisüberwachungsgesetz vorgesehen? Müsste der PüW dann nicht auch in allen anderen Gremien, die schweizweite Tarife erarbeiten, Einsitz nehmen?

Saxer: Der Vorschlag, dem Preisüberwacher in TARMED Suisse einen Beobachterstatus einzuräumen, ist ordnungspolitisch verfehlt. Er stammt aus der Mottenkiste eines kooperativen Staatsinterventionismus und hat mit einer rechtsstaatlich sauberen und klaren Rollenverteilung zwischen den verschiedenen Akteuren im Gesundheitswesen wenig zu tun. Natürlich möchte die Politik im Gesundheitswesen endlich Erfolge vorweisen können. Derzeit definiert die Politik den Begriff «Erfolg» inhaltlich einseitig und dies deutlich: Statt einer qualitativ hochstehenden und umfassenden Gesundheitsversorgung für alle setzt die Politik auf Kostensenkungen und Sparen, was angesichts der gesellschaftlichen Entwicklungen (Demographie, Bevölkerungswachstum, technologische Entwicklungen usw.) kein erfolgversprechendes Ziel sein kann. Dessen ungeachtet sollen nun die Tarifpartner an den Händen geführt werden, um sich auf politisch vorgegebene Sparziele zu einigen. Wenn dies nicht gelingt, soll die Regierung verbindlich entscheiden können. Die Preisüberwachung soll damit von einer wettbe- werbspolitischen Fachbehörde zum Erfüllungsgehilfen einer bundesrätlichen Sparpolitik mutieren.

Das Gesetz sieht ein Primat von Verhandlungslösungen der Tarifpartner vor, mit einer anschliessenden Genehmigung des Verhandlungsresultats durch die Exekutive im Licht und in Anwendung der Tarifgrundsätze von KVG und KVV. Verhandlungen sind oft mühsam. Geprägt von Interessengegensätzen dauert es häufig länger, bis es zu einer Einigung kommt. Dies liegt in der Natur der Sache, vor allem im Gesundheitswesen. Wenn indes, wie von der EFK vorgeschlagen, der Bundesrat bei fehlender Einigung der Tarifpartner eine vorläufige Lösung dekretieren kann, werden Verhandlungen von der Einschätzung der Verhandlungspartner ge-

\section{«Der Vorschlag, dem Preisüber- wacher in TARMED Suisse einen Beobachterstatus einzuräumen, ist ordnungspolitisch verfehlt»}

prägt sein, wer am ehesten ein bundesrätliches Dekret in seinem Sinne erwarten kann. Damit sind freie Verhandlungen nicht mehr möglich, sondern es dominiert die Beurteilung der eigenen Verhandlungsposition mit Blick auf einen möglichen bundesrätlichen Entscheid. Kann sich eine anschliessende Verhandlungslösung wesentlich vom Dekret des Bundesrats, der ja die Lösung wiederum zu genehmigen hat, entfernen? Die Antwort ist ebenso eindeutig wie ernüchternd und lautet, wenig überraschend - nein.

Der Vorschlag der EFK würde die TARMED-SuissePartner zwingen, ihre Grundverträge anzupassen. Dies wäre ein wesentlicher Eingriff in ihre Tarifautonomie. Ferner würde der Erfolg von Verhandlungen zwischen den Tarifpartnern von der Geduld des Zuchtmeisters, eben des Preisüberwachers, abhängen. Dieser wäre nicht blosser Beobachter, sondern massgebender Akteur, weil er mittels Fristansetzung das Verhandlungstempo diktieren und nach Fristablauf den Fall zur Entscheidung dem Bundesrat überweisen kann. Zugleich spielt der Preisüberwacher mit seinen Empfehlungen eine entscheidende Rolle bei den Tarifverträgen. Als empfehlendes Fachgremium und als Hilfsorgan des Entscheidgremiums Bundesrat hätte er also eine Doppelrolle. Diese ist gesetzlich nicht vorgesehen, und die Einsitznahme in Entscheidgremien von Wirtschaftssubjekten, deren Tätigkeit zugleich von ihr beobachtet wird, ist mit gutem Grund keine gesetzliche Aufgabe der Preisüberwachung. Rechtsstaatlich kann die fehlende gesetzliche Grundlage nicht einfach mit einem politisch erzwungenen Konsens der TARMED-Suisse-Partner kompensiert werden. Bei behördlich festgesetzten oder genehmigten Preisen hat der Preisüberwacher ein vorgängiges 
Empfehlungsrecht. Dabei soll es bleiben. Ein Sonderregime im Krankenversicherungsbereich wäre auch aus Gründen der Rechtsgleichheit nicht akzeptabel.

\section{Frage an Dr. oec. HSG Willy Oggier}

Die EFK zitiert Art. 59c im Zusammenhang mit der Tarifstruktur TARMED. Sie haben erst kürzlich zusammen mit Herrn Professor Saxer ein Gutachten zu Art. 59c verfasst. Gehört die Tarifstruktur TARMED in die Zuständigkeit von Art. 59c? Bezieht sich der Artikel nicht vielmehr auf den Tarif-Taxpunktwert zur Tarifstruktur sowie die restlichen Bedingungen eines Tarifvertrages? Ist nicht der Artikel 43 Abs. 4 KVG diesem Verordnungsartikel übergeordnet? Oggier: Gesundheitsökonomisch betrachtet, stützt sich der Bericht der Eidgenössischen Finanzkontrolle (EFK) zu stark auf die Wirtschaftlichkeit ab. Denn es geht nicht nur um die Frage, ob Art. 43 Abs. 4 KVG Art. 59c KVV übergeordnet ist. Die EFK selbst verweist in ihrem Bericht (Seite 20) auf Art. 46 Abs. 4 KVG, wonach die Genehmigungsbehörde prüft, ob der Tarifvertrag mit dem Gesetz und dem Gebot der Wirtschaftlichkeit und Billigkeit in Einklang steht. Gemäss Art. 32 Abs. $1 \mathrm{KVG}$, einem der Schlüsselartikel des Gesetzes, geht es nicht nur um die Wirtschaftlichkeit, sondern um die Wirksamkeit, Zweckmässigkeit und Wirtschaftlichkeit. Man beachte bitte die aus gesundheitsökonomischer Sicht korrekte Reihenfolge.

\section{«Was wir für die Zukunft benöti- gen, ist eine vermehrte Beachtung der Aspekte Wirksamkeit und Zweckmässigkeit»}

Wo das eigentliche Problem zu orten ist, zeigt die EFK auf (Seite 81): «Gemäss Angaben FMH sind ca. 40 Leistungen heute ohne Tarif und diese werden wahrscheinlich mittels Analogieposition verrechnet.» Oder in anderen Worten: Für gesetzliche Pflichtleistungen gibt es die gesetzlich vorgeschriebenen Tarifpositionen nicht. Es darf daher nicht erstaunen, dass Unternehmer versuchen werden, ihren angefallenen Aufwand anderweitig verrechnen zu können. Wenn dem so ist, kann aber ein Kostenvergleich zwischen verschiedenen Ärzten und selbst von einzelnen Ärzten zwischen verschiedenen Jahren nicht seriös durchgeführt werden, weil riskiert wird, Äpfel mit Birnen zu vergleichen. Denn es dürfte davon auszugehen sein, dass die Anzahl Leistungen ohne Tarif im Zeitablauf angesichts der untauglichen Entscheidungsstrukturen bei den TARMED-Gremien (Prinzip der Einstimmigkeit) zunehmen dürfte.

Was wir für die Zukunft benötigen, ist daher eine vermehrte Beachtung der Aspekte Wirksamkeit und Zweckmässigkeit. Dazu braucht es Daten, und ohne diese kann die Wirtschaftlichkeit gesundheitsökonomisch auch nicht sinnvoll analysiert werden. Denn das Ziel der Wirtschaftlichkeit ist immer ein sekundäres, instrumentelles Ziel, das erst nach Definition des primären Ziels, der Wirksamkeit, sinnvoll erörtert werden kann.

Dem Gedanken der Wirtschaftlichkeit trägt die Eidgenössische Preisüberwachung heute Rechnung. Das ist gemäss Preisüberwachungsgesetz auch ihre Aufgabe. Für die Beurteilung der Kostenneutralität und von Art. 59c KVV braucht es aber vorgängig eine Beurteilung der Wirksamkeit und Zweckmässigkeit. Dies kann nicht Aufgabe der Preisüberwachung sein, sondern müsste durch andere Akteure erfolgen. Wenn die Tarifparteien dazu nicht in der Lage sind, müsste dies subsidiär durch den Staat geschehen. Damit wäre auch die Grundlage für eine sinnvolle Güterabwägung zwischen den drei Kriterien Wirksamkeit, Zweckmässigkeit und Wirtschaftlichkeit gegeben. Denn Medizin funktioniert in der Regel nicht nach dem Zufalls-, sondern nach dem Zuweisungsprinzip. Nur im ersten Fall wäre aber die starke Betonung der Wirtschaftlichkeit ohne Prüfung von Wirksamkeit und Zweckmässigkeit unter bestimmten Rahmenbedingungen vertretbar. Das Zuweisungsprinzip lässt es dagegen als wahrscheinlich erscheinen, dass die Morbiditätsverteilung unter den Leistungserbringern unterschiedlich ausfällt.

Gerade in dieser Hinsicht haben wir in der Schweiz heute wohl die grössten Lücken. Wir wissen beispielsweise nicht einmal, wie viele Diabetiker es in der Schweiz gibt. Genau deswegen brauchen wir national einheitliche Krebs- und andere Register und die morbiditätsorientierte Verfeinerung des Risikoausgleichs unter den Krankenversicherern. Denn dann haben diese auch vermehrt ein Interesse an Wirksamkeits- und Zweckmässigkeits-Fragen und nicht nur an verzerrten Wirtschaftlichkeits-Vergleichen. Aus diesem Grund sollte die morbiditätsorientierte Verfeinerung des Risikoausgleichs auch in der laufenden politischen KVG-Debatte von der Vorlage zur integrierten Versorgung getrennt werden. Denn sonst läuft diese Verfeinerung Gefahr, zusammen mit der Vorlage zur integrierten Versorgung vom Souverän bachab geschickt zu werden. Dafür ist die Verfeinerung des Risikoausgleichs aber zu wichtig, denn es geht dabei um die Wirksamkeit, Zweckmässigkeit und Wirtschaftlichkeit der Behandlungen aller Grundversicherten und nicht nur um jene in Modellen der integrierten Versorgung.

\section{Frage an lic. iur. Peter Meier}

Sie haben ja die Vertragsentwicklung zwischen den Parteien sowohl den Rahmenvertrag als dann später auch die Anschlussverträge begleitet. Uns scheint, im Bericht der EFK werde Tarifstruktur TARMED und der KVG-Tarif beliebig miteinander vermischt und insbesondere würden die Zuständigkeiten beliebig gemischt. Klären Sie uns bitte auf: 
Wer ist für was zuständig und mit welcher Aufgabe? Was bedeutet ein Eingriff des Bundes in die Tarifautonomie der vier Partner, wie er von der EFK vorgeschlagen wird? Meier: Mit ihren Schlussfolgerungen versucht die EFK meines Erachtens, das Grundprinzip des KVGs in Sachen Tarife, nämlich das Prinzip der Vertragsfreiheit, auszuhebeln. Schon in der Botschaft zum KVG im Jahr 1991 (!) wurde ganz klar folgende Kompetenzordnung gefordert: Die Tarifstruktur sollte beim Einzelleistungstarif auf gesamtschweizerischer Ebene einheitlich festgelegt werden, und zwar durch Vereinbarung zwischen den Tarifpartnern. Die Festlegung der Taxpunktwerte dagegen sollte, mit Rücksicht auf die regionalen Kostenunterschiede, weiterhin durch dezentrale Vereinbarungen erfolgen.

«Sollten wir tatsächlich bei einem

Amtstarif landen, ist es um die Selbständigkeit der Ärzteschaft geschehen»

Diesem Grundgedanken haben die Tarifpartner Rechnung getragen. Auf der Ärzteseite hat die FMH die Federführung bei der Tarifstruktur übernommen und zusammen mit den Vertragspartnern den Rahmenvertrag TARMED mit den entsprechenden Anhängen ausgehandelt.

Infolge der Kompetenzen der kantonalen Ärztegesellschaften bezüglich der Taxpunktwerte, wurde die G7, als Vorgängerin der KKA, gegründet, welche die kantonalen Anschlussverträge und insbesondere die Taxpunktwerte mit den Vertragspartnern aushandelte. Wenn nun die EFK dem Bund die Kompetenzen für provisorische Massnahmen im Tarifwesen übertragen will, müssen sich diese logischerweise auf die Tarifstruktur und den Taxpunktwert, der ja den Preis der ärztlichen Leistung ergibt, beziehen. Das bedeutet, dass die eben erwähnte Vertragsautonomie durchbrochen wird und die Kompetenzen der Kantonalverbände und der kantonalen Behörden (Genehmigung) eingeschränkt oder aufgehoben werden sollen.

Natürlich waren dem Bundesrat (und übrigens auch der Preisüberwachung) die historisch bedingten unterschiedlichen Taxpunktwerte zwischen den Kantonen, aber auch innerhalb der Kantone, immer ein Dorn im Auge.

Im weiteren, und das ist für mich als Jurist von ganz entscheidender Bedeutung, gäbe es für den Fall von bundesrätlichen provisorischen Massnahmen im Tarifwesen keine Rechtsmittel. Ich habe in den letzten Jahren und Jahrzehnten mit Sorge die immer weiterführenden Eingriffe in die Vertrags- und Tarifautonomie festgestellt. Angefangen hat dies mit dem Tarifschutz im KVG, der Rolle der Preisüberwachung, die einseitig auf die Wirtschaftlichkeit fokussiert ist, der Handhabung der «Behördlichen Tarife» durch den Bund (Analysenliste), der Verordnung der sog. Tarifgestaltungsgrundsätze (u.a. Kostenneutralität), und jetzt sind wir bei der faktischen Forderung nach einem Amtstarif, wenn auch unter dem Deckmantel von provisorischen Tarifmassnahmen.

Sollten wir tatsächlich bei einem Amtstarif landen, ist es um die Selbständigkeit der Ärzteschaft geschehen. FMH und Kantonale Ärztegesellschaften, aber auch Berufsverbände, können dann als Gewerkschaften auftreten und ihre Forderungen in gewerkschaftlicher Manier durchzusetzen versuchen.

Ich hoffe, dass ich als selbständiger Anwalt diesen Tag nicht mehr erlebe.

\section{Sie lesen gerade eine Zeitschrift des Schweizerischen Ärzteverlags EMH}

\section{Wussten Sie schon,}

- dass EMH ein Gemeinschaftsunternehmen der Verbindung der Schweizer Ärztinnen und Ärzte FMH und der Schwabe AG ist, dem mit Gründung 1488 ältesten Druck- und Verlagshaus der Welt?

- dass EMH mit insgesamt zehn Fachzeitschriften, einem umfangreichen Online-Angebot sowie einem wachsenden Buchprogramm der führende Verlag für medizinische Zeitschriften in der Schweiz ist?

- dass sämtliche bei EMH erscheinenden Zeitschriften offizielle Publikationsorgane der jeweils zuständigen medizinischen Fachorganisationen sind?

Wenn Sie mehr über EMH wissen möchten, finden Sie unter www.emh.ch weitere Informationen. 